Original

\title{
Influencia de los turnos de trabajo y las guardias nocturnas en la aparición del Síndrome de Burnout en médicos y enfermeras
}

\author{
Influence of shift work and night shifts in the onset of the Burnout Syndrome in \\ doctors and nurses
}

\author{
Ambar Deschamps Perdomo 1,4, Sahilyn B. Olivares Román ${ }^{2,4}$, Kelman Luis De la Rosa Zabala ${ }^{3,4}$, Ángel Asunsolo \\ del Barco 5 \\ 1. Fundación Jiménez Díaz. Madrid. España. \\ 2. Hospital Universitario Puerta de Hierro, Majadahonda. Majadahonda. España. \\ 3. Hospital Universitario de Móstoles. Móstoles. España. \\ 4. Escuela Nacional de Medicina del Trabajo. Unidad Docente I. Madrid. España. \\ 5. Universidad de Alcalá de Henares. Facultad Medicina. Área Medicina Preventiva y Salud Pública, Ciencias \\ Sanitarias y Medico Sociales. Alcalá de Henares (Madrid). España.
}

Recibido: 24-08-11

Aceptado: 02-09-11

\section{Correspondencia}

Sahilyn Beatriz Olivares Román

Isla de Córcega, 8 Piso 1-B

28039. Madrid. España.

Tfno: 697-994-509

Fax: 91 1-917-653 * Hospital

e-mail: lyn_olivares@hotmail.com

Resumen

La sociedad actual que funciona 24 horas al día, obliga a las organizaciones y en consecuencia a sus empleados a someterse a horarios de trabajo que van en contra del ritmo natural de la vida. El horario por turno y las guardias, fuera de las horas normales del día, es un tema que cobra importancia, ya que son muchas las implicaciones que esto trae como consecuencia en la salud física y mental de quienes lo realizan.

Objetivo: Analizar la evidencia científica existente la influencia de los turnos de trabajo y las guardias nocturnas en la aparición del síndrome de Burnout en médicos y enfermeras.

Método: Varias bases de datos han sido analizadas (Medline, Pubmed, Lilacs, Cochrane), con descriptores específicos y según criterios de inclusión se ha obtenido la bibliografía.

Resultados: Se localizaron 40 artículos. De los cuales, 16 (40\%) corresponden a estudios en enfermeras y 24 (60\%) sobre médicos, principalmente médicos en formación. Parece existir una relación de la influencia de los turnos de trabajo y las guardias nocturnas con la aparición del síndrome de Burnout, en médicos y enfermeras.

Conclusión: La identificación de los factores de riesgo psicosocial a los que pueden estar expuestos los médicos permitirá adoptar medidas preventivas, que pueden ser útiles para mejorar la salud y la calidad de vida de este colectivo profesional.

Med Segur Trab (Internet) 2011; 57 (224) 224-241

Palabras clave: Trabajo a turno, Trabajo Nocturno, Síndrome del quemado, trastornos del sueño. 


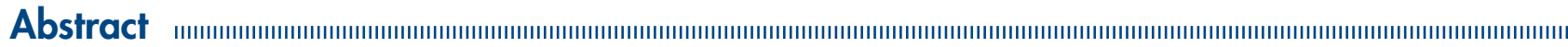

Society today works 24 hours a day, forcing organizations and their employees to submit work schedules that go against the natural rhythm of life. Shift work and night work, is an issue that is becoming important, as there are important consequences in physical and mental health of those who work this way.

Objective: Analyze the existing scientific evidence of the influence of shift work and night shifts in the onset of burnout syndrome among physicians and nurses.

Method: Several databases have been reviewed (Medline, Pubmed, Lilacs, Cochrane), with specific descriptors and bibliography has been obtained according to the criteria of inclusion.

Results: 40 articles were located, of which 16 (40\%) were studies of nurses and 24 (60\%) of physicians, mostly physicians studying their specialty. There seems to be a relationship of the influence of shift work and night shifts with the appearance of burnout syndrome in doctors and nurses.

Conclusions: The identification of psychosocial risk factors to which physicians may be exposed will allow us to take preventive measures that can be usefull to improve health and quality of life of this professional group.

Med Segur Trab (Internet) 2011; 57 (224) 224-241

Keywords: shift work, Night Work, Burnout Syndrome, Sleep Disorders. 


\section{INTRODUCCIÓN}

La sociedad actual que funciona 24 horas al día, obliga a las organizaciones y en consecuencia a sus empleados a someterse a horarios de trabajo que van en contra del ritmo natural de la vida. El horario por turno y las guardias, fuera de las horas normales del día, es un tema que cobra importancia, ya que son muchas las implicaciones que esto trae como consecuencia en la salud física y mental de quienes lo realizan ${ }^{1}$.

El trabajo por turno se ha convertido en una de las cuestiones laborales que más discrepancias y conflictos genera en las empresas. Dicho trabajador es aquel que presta sus servicios en horas diferentes, en un período determinado de días o de semanas. Es una forma de organización del trabajo en equipo, por la cual el trabajador ocupa sucesivamente el mismo puesto de trabajo, según un ritmo continuo o discontinuo, realizándose obligatoriamente de forma rotativa por los componentes del grupo laboral. Hay sectores como por ejemplo el sector salud, donde estos turnos de trabajo son muy prolongados o irregulares, llegando a afectar tanto la salud como la vida del trabajador.

La situación que se observa en muchas empresas entre las que destaca el sector sanitario, en especial en los grupos de médicos y enfermeras, trae consigo consecuencias negativas a distintos niveles tanto de productividad, atención al paciente, aumento del ausentismo, y quizá lo más delicado, efectos sobre el bienestar de los trabajadores, donde se evidencia cada vez con mayor prevalencia la aparición del Síndrome de Burnout (SB).

La población sanitaria no escapa de este problema ya que viven a diario con los turnos de trabajo. Waterhouse $\mathrm{JM}^{2}$ desde 1994 reportaba que los médicos jóvenes (residentes) trabajando tandas de 36 ó 48 horas podrían cometer errores debido a la fatiga. A través de los años algunos países han implantado turnos más cortos, pero el hecho de cambiar el ritmo natural sueño-vigilia afecta al trabajador aun con estas reducciones. Rebecca Smith Cogins, Moore-ede y otros ${ }^{3,4}$ han realizado estudios en los que se han demostrado que interrupciones crónicas del ritmo circadiano están asociadas a un mayor riesgos de desordenes de sueño-vigilia, problemas gastrointestinales y cardiovasculares.

El SB, conocido en la literatura de habla hispana como síndrome de Desgaste Profesional $^{5,6}$ y más recientemente como síndrome de "quemarse por el trabajo" 7 fue descrito por Freudenberger en los años setenta ${ }^{8}$ Aunque existen múltiples definiciones, la más conocida es la de Maslach y Jakcson, que lo caracteriza como la presencia de altos niveles de agotamiento emocional (AE) y despersonalización (DP) y una reducida realización personal (RP) ${ }^{9}$.Síndrome que por las cargas de trabajo y la privación del sueño, se presenta en el ámbito sanitario cuando fracasan los mecanismos compensatorios de adaptación ante situaciones laborales con un estrés sostenido. Se observa con mayor frecuencia en trabajos con un desajuste entre las demandas y los recursos; y especialmente en personas con unas expectativas idealistas que encuentran una realidad frustrante ${ }^{10}$.

En el desarrollo del síndrome de Burnout intervienen factores ambientales, culturales y personales ${ }^{11}$. Entre los factores ambientales se ha observado que los trabajadores del sector servicios y los profesionales sanitarios, en especial médicos y enfermeras, son los que presentan mayores prevalencias de Burnout entre las distintas profesiones, entre otras razones por los turnos de trabajo y las guardias a las que estos grupos se ven sometidos. Algunos autores han observado que las sociedades occidentales con altos niveles de competitividad pueden predisponer a desarrollar SB, otros consideran que no sólo implica a sociedades del bienestar y que es un problema transnacional y transcultural ${ }^{12}$.

La Comisión Europea, a través de la Fundación Europea para la Mejora de las Condiciones de Vida y Trabajo (1999) ha realizado un estudio sobre el estrés laboral en el que concluye que el 28\% de los trabajadores europeos padece estrés y el 20\% Burnout (se sienten "quemados" en su trabajo) ${ }^{13}$. Ésta problemática, cada vez es un punto de interés para realizar más estudios y buscar algunas metodologías que ayuden al personal que trabaja en horarios nocturnos. R Doug McEvoly y León L Falta postulan, que dormir 
la siesta durante un turno de noche puede beneficiar tanto a profesionales de la salud como a sus pacientes ${ }^{14}$.

Los turnos de trabajo, las guardias y los trastornos que se derivan a consecuencia de los mismos como es el síndrome de Burnout; en trabajadores que son sometidos a amplias jornadas laborales, hacen cada vez mayor la necesidad de conocer en detalle el problema y poder crear medidas que disminuyan sustancialmente las repercusiones negativas sobre la salud.

\section{OBJETIVOS}

El principal objetivo de este estudio es analizar la evidencia científica existente acerca de la influencia de los turnos de trabajo y las guardias nocturnas en la aparición del Síndrome de Burnout en médicos y enfermeras.

Como objetivos secundarios, se pretende:

- Identificar las consecuencias sobre la salud de los turnos de trabajo y las guardias en el personal médico y de enfermería.

- Estudiar la relación que guardan los turnos de trabajo y las guardias con el riesgo de accidente de tráfico y accidente en la labor hospitalaria.

\section{MATERIAL Y MÉTODOS}

La búsqueda de los trabajos de investigación se ha realizado en varias fuentes de datos, y ha sido desarrollada en dos etapas.

Las Bases de Datos bibliográficas y los términos de búsqueda (DESC) utilizados se muestran en la Tabla I.

Tabla 1. Tabla de búsqueda con los términos DECS

\begin{tabular}{|c|c|c|c|c|c|c|}
\hline Desc & Pubmed & $\begin{array}{c}\text { Google } \\
\text { Académico }\end{array}$ & $\begin{array}{l}\text { Biblioteca } \\
\text { Cochrane }\end{array}$ & Ilo.Org & Medline & Lilac \\
\hline Shift Work & 8,870 & $3,460,000$ & 204 & 2263 & 1 & 248 \\
\hline Night Work & 2,884 & $2,120,000$ & 167 & 1353 & 7 & 128 \\
\hline Work Hours & 11,746 & $3,990,000$ & 814 & 3893 & 25 & 434 \\
\hline Shift Worker & 3,403 & $1,710,000$ & 10 & 2167 & Cero & $25 / 3$ \\
\hline $\begin{array}{l}\text { Shift work } \\
\text { and night work }\end{array}$ & 1103 & 70 & 70 & 588 & Cero & 1214 \\
\hline $\begin{array}{l}\text { Shift work } \\
\text { and night work } \\
\text { and work hour }\end{array}$ & 284 & $\begin{array}{c}\text { De } 22 \\
\text { hallazgos/ } \\
1 \text { válidos }\end{array}$ & 8 & 535 & Cero & 512 \\
\hline $\begin{array}{l}\text { Shift work } \\
\text { and work hour } \\
\text { and physicians } \\
\text { Últimos } 10 \text { años }\end{array}$ & $\begin{array}{c}\text { De } 24 \\
\text { hallazgos/ } \\
3 \text { válidos }\end{array}$ & 519 & 3 & 166 & Cero & $\begin{array}{c}35 \\
\text { hallazgos/ } \\
19 \text { selec. }\end{array}$ \\
\hline $\begin{array}{l}\text { Shift work } \\
\text { and hour night }\end{array}$ & $\begin{array}{l}78 \text { hallazgos/ } \\
11 \text { válidos. }\end{array}$ & 381 & 8 & 535 & Cero & 512 \\
\hline $\begin{array}{l}\text { Shift work } \\
\text { and hour night } \\
\text { Últimos } 5 \text { años }\end{array}$ & & $\begin{array}{c}167 \text { hallazgos/ } \\
1 \text { selec. }\end{array}$ & 2 & 383 & Cero & - \\
\hline $\begin{array}{l}\text { Shift work } \\
\text { and work hour } \\
\text { and nurses }\end{array}$ & $\begin{array}{c}\text { De } 43 \\
\text { hallazgos/ } 6 \\
\text { válidos }\end{array}$ & 226 & 3 & 287 & Cero & 113 \\
\hline
\end{tabular}




\begin{tabular}{|c|c|c|c|c|c|c|}
\hline Desc & Pubmed & $\begin{array}{c}\text { Google } \\
\text { Académico }\end{array}$ & $\begin{array}{l}\text { Biblioteca } \\
\text { Cochrane }\end{array}$ & Ilo.Org & Medline & Lilac \\
\hline $\begin{array}{l}\text { Shift work } \\
\text { and work hour } \\
\text { and nurses } \\
\text { Últimos } 5 \text { años }\end{array}$ & & $\begin{array}{c}164 \\
\text { hallazgos/ } \\
1 \text { selec. }\end{array}$ & Cero & 201 & Cero & - \\
\hline $\begin{array}{l}\text { shift work } \\
\text { and night work } \\
\text { and sleep } \\
\text { disorders and } \\
\text { physicians }\end{array}$ & $\begin{array}{l}16 \text { hallazgos/ } \\
6 \text { válidos }\end{array}$ & $\begin{array}{l}37 \text { hallazgos/ } \\
1 \text { selec. }\end{array}$ & Cero & $\begin{array}{c}7 \text { hallazgo/6 } \\
\text { selec. }\end{array}$ & Cero & $\begin{array}{c}21 \text { hallazgos/ } \\
5 \text { selec. }\end{array}$ \\
\hline $\begin{array}{l}\text { shift work } \\
\text { and night work } \\
\text { and stress } \\
\text { and physicians }\end{array}$ & $\begin{array}{l}5 \text { hallazgos/ } \\
1 \text { válido }\end{array}$ & $\begin{array}{l}60 \text { hallazgos/ } \\
1 \text { selec. }\end{array}$ & Cero & 47 & Cero & $\begin{array}{c}7 \text { hallazgos/ } \\
5 \text { selec. }\end{array}$ \\
\hline $\begin{array}{l}\text { Shitf work } \\
\text { and sindrome } \\
\text { burnout }\end{array}$ & $\begin{array}{c}\text { De hallazgos } \\
10-10 / \\
\text { válido } 19\end{array}$ & 21,900 & Cero & $\begin{array}{c}6 \text { hallazgos/ } \\
5 \text { selec. }\end{array}$ & Cero & Cero \\
\hline $\begin{array}{l}\text { shift work } \\
\text { and syndrome } \\
\text { burn out } \\
\text { and physicians }\end{array}$ & - & $\begin{array}{l}16 \text { hallazgos/ } \\
5 \text { selec. }\end{array}$ & Cero & $\begin{array}{l}8 \text { hallazgo/ } \\
5 \text { selec./ }\end{array}$ & Cero & $\begin{array}{c}1 \text { hallazgo/ } \\
1 \text { selec. }\end{array}$ \\
\hline $\begin{array}{l}\text { shift work } \\
\text { and syndrome } \\
\text { burn out } \\
\text { and nurses }\end{array}$ & - & 26 & Cero & $\begin{array}{c}10 \text { hallazgo/ } \\
8 \text { selec. }\end{array}$ & Cero & Cero \\
\hline
\end{tabular}

La primera fase del proceso, se basó en elegir los artículos investigación científica que cumplan los criterios de inclusión. Artículos que tratan sobre los turnos de trabajo de médicos y enfermeras, del desarrollo del Síndrome de Burnout, de los accidentes de tráfico o accidentes laborales, y sobre los efectos en la salud del trabajador a turno nocturno o rotatorio. Se incluyen 130 resúmenes publicados desde el 1990 hasta la fecha, redactados en los idiomas español y/o inglés.

La segunda fase del proceso de selección, se realiza después de leer los textos completos, de los 130 artículos anteriormente seleccionados y se procedió a implementar los criterios de exclusión. Se descarta aquellos trabajos cuyo resultado no sea estadísticamente significativo y los que no se recuperen a texto completo.

Finalmente se incluyeron en el estudio un total de 27 artículos a texto completo. De los artículos excluidos para el estudio, 22 se excluyeron porque no obtenían resultados estadísticamente significativos, 12 no cumplían con los idiomas requeridos, 3 de ellos tenían fecha de publicación fuera del rango del periodo de estudio y el resto no coincidían con el tema central o no se pudo obtener los textos completos de los artículos.

\section{RESULTADOS}

Se procedió a estudiar los turnos de trabajo y las guardias, tanto en el personal médico como en el personal de enfermería del sector sanitario; pero como los mismos aunque trabajan con horarios por turnos no trabajan de corrido las mismas cantidades de horas, y para no incurrir en un sesgo se prefirió analizar los resultados por separado. 


\section{Resultados obtenidos en el personal médico}

\subsection{Aparición del Síndrome de Burnout}

En la tabla 2 se presentan un total de 9 artículos que relacionan el Síndrome de desgaste profesional (Burnout) con los turnos de trabajo y las guardias en los médicos, especialmente en los médicos en formación o médico residente; por ser este el grupo más ampliamente estudiado.

En 2001, en el Centros de Salud Torrelodones y San Lorenzo de El Escorial, Madrid, se realizo un estudio sobre la prevalencia y factores asociados al Burnout, donde se destacaron considerables niveles: un $30,6 \%$ dentro del rango alto para la subescala de cansancio emocional y con mayores puntuaciones en los varones ( $p=0,026)$. Un 43,9\% considera presentar o haber presentado algún tipo de alteración física o psíquica relacionada directamente con el desarrollo de su profesión, y el 38,3\% asocia directamente su desgaste con el exceso de demanda soportado habitualmente en sus consultas ${ }^{15}$.

Los médicos tienen una salud física mejor, pero una peor salud mental que la población general. Así mismo, la prevalencia de tentativa de suicidio, sobre todo en las especialidades médicas es mayor que la que presenta la población general. Pudiéndose explicar en gran medida, por el tipo de trabajo realizado que implica estar expuesto a un importante número de factores de riesgo de tipo psicosocial; como el contacto con el sufrimiento y la muerte, la falta de recursos materiales, las largas jornadas de trabajo, los horarios irregulares, la falta de recompensas profesionales, etc. ${ }^{16}$

La prevalencia del burnout es mayor en España y Argentina; y los profesionales que más lo padecen son los médicos. Pero existen variables protectoras como son el optimismo, la percepción de sentirse valorado, la satisfacción laboral, la valoración económica, entre otras ${ }^{17}$. Al igual que en el estudio de MA Sánchez et al. ${ }^{18}$ que revela que los médicos españoles, específicamente cirujanos ortopédicos, han demostrado niveles superior de desgaste profesional frente a los encontrados en otros países. Además, de que la prevalencia del síndrome de Burnout es superior en el ámbito público versus el privado.

Algunos autores junto con $\mathrm{J}_{\text {Cebriá }}{ }^{19}$ se limitaron a estudiar en particular de los médicos de familia, en un estudio longitudinal durante cinco años en ciudad de Barcelona. Donde se evidencio que dicho síndrome de Burnout ha aumentado, pues afecta a profesionales que anteriormente no padecían el síndrome, mientras que los médicos ya afectados tendían a cierta estabilidad.

M. Fonseca et al. ${ }^{20}$ ha asociado al Síndrome de burnout de forma significativa al hecho de realizar más de 4 guardias mensuales. Donde reveló que el $68.7 \%$ de los residentes de un hospital universitario en estudio estaban quemados en el trabajo. Haciendo mención a que los residentes de medicina interna y los de especialidades médicas son los que presentan una mayor tendencia. Sin embargo, estudios como el de O. Fernández Martínez et al. ${ }^{21}$ refieren que no está estadísticamente comprobado que el número de guardias influya. Sí determinaron que el $93 \%$ de los residentes presento un estado de quemado, haciendo notar que los que presentaron un alto nivel de despersonalización, no libraban las guardias.

En un estudio realizado en el Hospital Gregorio Marañón, por Casado Moragón et al ${ }^{22}$, se encontró diferencias significativas en cuanto a la presencia del síndrome de Burnout según el sexo del médico residente, según su edad o el estado civil y no según la antigüedad profesional. En este centro de salud las aéreas más afectadas fueron: urgencia, seguido de intensivo y paliativo. Mientras que en el 2009 Frade Mera et al. ${ }^{23}$ realizo un estudio comparativo en las unidades de cuidado intensivo, obteniendo una prevalencia del Burnout de un $14 \%$. Los resultados de este estudio en cuanto a la distribución por otras profesiones sanitarias, fueron que dentro del personal de enfermería, el 16\% presentaron el Síndrome de Burnout, entre los médicos residentes se vio un 14\%, entre los médicos no residentes, un $13 \%$ y entre los auxiliares un $10 \%$. 
Tabla 2. Aparición del Síndrome de Burnout en el personal médico por los turnos de trabajo y las guardias

\begin{tabular}{|c|c|c|c|}
\hline N. ${ }^{\circ}$ & Autor & Pub. & Tipo de estudic \\
\hline 1 & $\begin{array}{l}\text { M.A. Caballero } \\
\text { Martín, F. } \\
\text { Bermejo } \\
\text { Fernández, R. } \\
\text { Nieto Gómez } \\
\text { y F. Caballero } \\
\text { Martínez } 15\end{array}$ & 2001 & $\begin{array}{l}\text { Transversal, } \\
\text { descriptive }\end{array}$ \\
\hline 2 & $\begin{array}{l}\text { Escribà-Agüir } \\
\text { V, Artazcoza L, } \\
\text { Pérez-Hoyosa } \\
\text { S.16 }\end{array}$ & 2008 & Transversal \\
\hline 3 & $\begin{array}{l}\text { Armand } \\
\text { Grau, Daniel } \\
\text { Flichtentrei, Rosa } \\
\text { Suñer, María } \\
\text { Prats y Florencia } \\
\text { Braga } 17\end{array}$ & 2007 & Transversal \\
\hline 4 & $\begin{array}{l}\text { Sánchez- Madrid } \\
\text { MA, Delgado } \\
\text { Martínez AD, } \\
\text { Alcalde Pérez } \\
\text { D18 }\end{array}$ & 2005 & $\begin{array}{l}\text { Descriptivo } \\
\text { Transversal }\end{array}$ \\
\hline
\end{tabular}

5 Cebriá J, Palma

2008 Estudio C, Sobrequés J, Ger S, Ferrer M, Segura J.19

Detectaron niveles de este síndrome preocupantemente elevados entre nuestros profesionales, similares a los encontrados en otras encuestas nacionales. Los encuestados asocian su malestar con algunos factores laborales que podrían ser fácilmente modificables. La formación recibida en cuanto a técnicas de auto-control y manejo del estrés son claramente insuficientes.

El ambiente psicosocial y la satisfacción laboral influyen negativamente en el síndrome de burnout, y afectan a las dimensiones de cansancio emocional y despersonalización. En el cual la prevalencia de alta despersonalización es mayor que la observada en otros estudios de médicos especialistas de otros países, así como en trabajadores sanitarios de hospitales y en médicos de atención primaria del Estado español.

La prevalencia del Burnout es mayor en España y Argentina y los profesionales que más lo padecen son los médicos. La edad, tener hijos, la percepción de sentirse valorado, el optimismo, la satisfacción laboral y la valoración económica, son variables protectoras de burnout.

Se determinó el grado de desgaste profesional en los cirujanos ortopédicos de España y la influencia de diversos factores sobre éste. Donde se obtuvo que un $44.1 \%$ de los participantes presentó un alto grado de desgate profesional por cansancio, un $64,6 \%$ en despersonalización y un $24,5 \%$ por baja realización personal. Dicta que tener varios hijos, un contrato fijo y trabajar en sector privado son factores que se asocian con mejores puntuaciones en las escalas de Burnout.

Se analizó la evolución del síndrome de Burnout mediante el seguimiento de un grupo de médico de familia en la ciudad de Barcelona a lo largo de cinco años. Se determinó que los niveles de desgaste profesional en los médicos de familia se han incrementado globalmente en cinco años.

6 Fonseca M, Sanclemente G, Hernandez C, Visiedo C, Bragulat E, Miró O. 20

Se investigó el grado de burnout entre los residentes de un hospital universitario y los factores asociados al mismo. Obteniéndose que más de la mitad de los residentes estaban quemados en el trabajo, específicamente el $68,7 \%$ de los residentes. Donde el hecho de realizar 5 o más guardias influía forma directa significativa, asociándose con el cansancio emocional pero no con la despersonalización ni con el grado de realización personal. Se observó una mayor tendencia entre los residentes de medicina interna y especialidades médicas con respecto al resto de residentes.

7 Fernández

2007 Descriptivo Transversal
Martínez $\mathrm{O}$

Hidalgo Cabrera

C, Martin Tapia

A, Montero Suarez S, García del Rio García B.21
Comenta sobre el grado de desgaste profesional que médicos residentes han desarrollado en un hospital general. Donde el 93\% de los residentes presentó Burnout y se encontró un alto Burnout en todos los residentes que no libraban las guardias. Sin haber pruebas estadísticamente significativas que relacionaran el nivel de quemarse con el sexo, la edad, los años de residencia, con la especialidad, ni con el número de guardias. 


\begin{tabular}{|c|c|c|c|c|}
\hline N. ${ }^{o}$ & Autor & Pub. & Tipo de estudio & Resumen \\
\hline 8 & $\begin{array}{l}\text { Casado } \\
\text { Moragón A, } \\
\text { Muñoz Duran } \\
\text { G, Ortega } \\
\text { González N, } \\
\text { Castellanos } \\
\text { Asenjo A, } \\
\text { Rodríguez } \\
\text { Blázquez ME, } \\
\text { García Aroca, } \\
\text { Concha } 22\end{array}$ & 2005 & $\begin{array}{l}\text { Retrospectivo- } \\
\text { Transversal }\end{array}$ & $\begin{array}{l}\text { Se determinó la influencia de factores sociodemográficos } \\
\text { y laborales en la prevalencia del síndrome de burnout del } \\
\text { personal sanitario del Hospital Gregorio Marañón. Los } \\
\text { niveles más altos de burnout se observaron en urgen- } \\
\text { cias, seguidos de intensivos y paliativos. Se observaron } \\
\text { diferencias significativas en burnout según sexo, edad y } \\
\text { estado civil entre trabajadores de los tres servicios. }\end{array}$ \\
\hline 9 & $\begin{array}{l}\text { Frade Mera MJ, } \\
\text { Vinagre Gaspar } \\
\text { R, Zaragoza } \\
\text { García I, Viñas } \\
\text { Sánchez S, } \\
\text { Antúnez Melero } \\
\text { E, Álvarez } \\
\text { González S, et } \\
\text { al } 23\end{array}$ & 2009 & Analítico & $\begin{array}{l}\text { Se determinó la prevalencia del síndrome de Burnout en } \\
\text { el personal sanitario de distintas unidades de cuidados } \\
\text { intensivos, compara sus dimensiones y variables socio } \\
\text { demográficas laborales. Con una prevalencia del Burnout } \\
\text { de un } 14 \% \text {. Siendo el personal de enfermería el más afec- } \\
\text { tado. Además de comentar que este síndrome se asocia a } \\
\text { una baja satisfacción profesional, a una relación regular } \\
\text { con compañeros, a un bajo rendimiento laboral y a un } \\
\text { elevado cansancio emocional. }\end{array}$ \\
\hline
\end{tabular}

\subsection{Accidente de tráfico y accidentes en el trabajo hospitalario a consecuencia de los largos turnos de trabajo}

La valoración de la tasa de accidentes automovilísticos que el trabajador médico presenta tras su turno nocturno está muy discutida y de igual forma, las posibles alteraciones de su rendimiento profesional y su repercusión en la salud del paciente. Cinco (5) artículos de los revisados hacen mención a este tema, coincidiendo en que existe una tasa mayor de accidentes automovilísticos en aquellos médicos que trabajan 24 horas o más. En el ensayo de Lockley et al. ${ }^{24}$ se obtuvo que los médicos residentes que habían trabajado 24 horas o más tenían 2.3 veces más probabilidades de sufrir un accidente automovilístico después de su jornada en comparación con los que trabajaron menos de 24 horas, con un riesgo mensual de un accidente mayor en un 16,2\%.

En el estudio descriptivo de E.J. Olson et al. ${ }^{25}$ comentan que aunque hay evidencias de que la pérdida de sueño aumente los accidentes automovilísticos en los médicos, aún no ha sido completamente aclarado que también se altere la seguridad del paciente. El Consejo de Acreditación de la Educación Médica para Graduados (ACGME) ha respondido a las crecientes preocupaciones sobre la pérdida del sueño de los médicos en formación. Los opositores, argumentan que los límites de horas de trabajo amenazan profesionalidad de los residentes, la educación y la prestación de la atención al paciente,. Los proponentes, argumentan límites más estrictos. Mientras que los médicos residentes tienen opiniones discordantes sobre los límites de horas de trabajo en la atención al paciente y la educación, pero los datos hasta ahora, no revelan, cambios importantes en la mortalidad de los pacientes según las nuevas normas.

Sin embargo, B. J. Wilhelm y A. Widman en el 2009 estudiaron 34 médicos a través de la prueba Pupillographic Somnolencia (PST) y una escala sugestiva en la primera mitad del día, para valorar la seguridad del trabajador tras un turno de 24 horas. Donde el lnPUI era más grande después de turno de noche, que es el análisis estadístico del logaritmo natural que se utiliza para estudiar el índice distribución pupilar en la PST. Con una Diferencia N-D: medina 0,19 , min.-0,71, máx. $1.29, p=0.03$. Correlacionando las medidas objetivas con los resultados subjetivos practicados. Evidenciándose que los trabajadores de turno nocturno presentan una reducción en la seguridad frente al pacientes y frente a su propia seguridad, cuando conduce de regreso a casa ${ }^{26}$. 
De una encuesta telefónica con marcación digital al azar, se obtuvieron 1,200 encuestas completas en la población de los EEUU, a fin de obtener una perspectiva de la opinión pública sobre reducir las horas de trabajo del médico residente. El 81\% (IC 95\%: $79 \%$ a $84 \%$ ) piensa que una reducción de las horas de trabajo del médico residente sería muy o bastante eficaz para reducir los errores médicos. En comparación con el 1\% que opina que debería permitírsele al médico residente trabajar más de 24 horas consecutivas ${ }^{27}$.

En el estudio prospectivo de Rebecca Smith-Coggins se demostró que el médico tras trabajar 3 noches consecutivas vs 3 días consecutivos presentaba una disminución de su rendimiento, por ejemplo eran más lentos en la intubación de un maniquí. Sin embargo, también es cierto, que el médico en las últimas horas de la guardia presenta cierta tendencia a la reactivación, por lo que al requerir intubar al maniquí eran más rápidos ${ }^{28}$.

Tabla 3. Accidentes de tráfico y accidentes en el trabajo hospitalario a consecuencias de los largos turnos de trabajo

\begin{tabular}{|c|c|c|c|c|c|}
\hline N. ${ }^{\circ}$ & Autor & Pub. & Tipo de estudio & Idioma & Resumen \\
\hline 1 & $\begin{array}{l}\text { Lockley SW; } \\
\text { Landrigan CP; } \\
\text { Barger LK; } 24\end{array}$ & $\begin{array}{c}\text { Ago } \\
2006\end{array}$ & $\begin{array}{l}\text { Ensayo } \\
\text { aleatorio de } \\
\text { cohort }\end{array}$ & Inglés & $\begin{array}{l}\text { Controversia sobre } \\
\text { el horario óptimo } \\
\text { para los médicos en } \\
\text { formación. Evaluando } \\
\text { los efectos de las horas } \\
\text { de trabajo en el sueño, } \\
\text { la salud y la seguridad } \\
\text { del residente y sus } \\
\text { pacientes. }\end{array}$ \\
\hline
\end{tabular}
$\begin{array}{llll}2 & \text { Olson EJ; Drage } & \text { Nov } & \text { Descriptivo- } \\ \text { LA; Auger RR.25 } & 2009 & \text { Revisión }\end{array}$
Descripción básica de los procesos de regulación del sueño, del impacto de los horarios, las pruebas de laboratorio que demuestran efectos en los médicos y los efectos personales por dicha pérdida de sueño.
3 Wilhelm BJ; Widmann A; Durst W; Heine C; Otto G 26
Jun Experimental Inglés 2009

Estudio objetivo sobre la discusión de la seguridad de los médicos tras los turnos de noche. Utilizan do la prueba Pupillographic Somnolencia (PST).

\begin{tabular}{l}
\multicolumn{1}{c}{ Resultado } \\
Los residentes que \\
habían trabajado \\
24 horas o más \\
presentaban 2.3 veces \\
más probabilidades \\
de sufrir un accidente \\
automovilístico. \\
También se encontró \\
que pre- sentaban falta \\
de atención, con un \\
36\% de errores \\
médicos serios. \\
Cada vez hay más \\
evidencia sólida que \\
demuestra que la \\
pérdida de sueño \\
disminuye la realización \\
del médico y el \\
bienestar. Los turnos de \\
trabajo prolongados se \\
asocian con más errores \\
médicos, falta de \\
atención, rendimiento \\
cognitivo y un aumento \\
en la frecuencia de los \\
accidentes tráfico. de \\
vehículos. Sin embargo \\
no está comprobado \\
que afecte la seguridad \\
del paciente. \\
A pesa de
\end{tabular}
A pesar de una media de $4 \mathrm{~h}$ de sueño el médico presenta somnolencia considerable, lo que implica reducir la seguridad tanto para los pacientes; si continúan trabajando, como para los mismos médicos, mientras viaja a casa.




\begin{tabular}{|c|c|c|c|c|c|c|}
\hline N. ${ }^{\circ}$ & Autor & Pub. & Tipo de estudio & Idioma & Resumen & Resultado \\
\hline 4 & $\begin{array}{l}\text { Blum AB; } \\
\text { Raiszadeh F; } \\
\text { Shea S; Mermin } \\
\text { D; Lurie P; } \\
\text { Landrigan CP; } \\
\text { Czeisler CA. } 27\end{array}$ & 2010 & $\begin{array}{l}\text { Descriptivo } \\
\text { Encuesta }\end{array}$ & Inglés & $\begin{array}{l}\text { Evaluar la perspectiva } \\
\text { del público americano } \\
\text { sobre la propuesta del } \\
\text { Instituto de Medicina } \\
\text { (IOM) de reducir las } \\
\text { horas de trabajo del } \\
\text { médico residente } \\
\text { debido al incremento } \\
\text { de errores médicos, } \\
\text { falta de atención y } \\
\text { accidente de tráfico. } \\
\text { Mediante una encuesta } \\
\text { nacional por teléfono } \\
\text { de marcación digital } \\
\text { al azar. }\end{array}$ & $\begin{array}{l}\text { El público } \\
\text { estadounidense } \\
\text { favorece la propuesta } \\
\text { de la OIM de la } \\
\text { suspensión de los } \\
\text { turnos de } 30 \text { horas } \\
\text { sin dormir. Con un } \\
\text { total del } 81 \% \text { (IC } \\
95 \% \text { : } 79 \% \text { a } 84 \% \text { cree } \\
\text { que la reducción de } \\
\text { las horas de trabajo } \\
\text { médico residente } \\
\text { sería muy o bastante } \\
\text { eficaz para reducir los } \\
\text { errores médicos, y el } \\
68 \% \text { (IC } 95 \%: 65 \% \text { a } \\
71 \% \text { ) favorece de la } \\
\text { propuesta de la OIM } \\
\text { de no más de } 16 \text { horas } \\
\text { seguidas de trabajo. }\end{array}$ \\
\hline 5 & $\begin{array}{l}\text { Smith-Coggins } \\
\text { R; Rosekind } \\
\text { MR; Buccino } \\
\text { KR; Dinges DF; } \\
\text { Moser RP } 28\end{array}$ & $\begin{array}{l}\text { Oct } \\
1997\end{array}$ & $\begin{array}{l}\text { Estudio } \\
\text { prospectivo, } \\
\text { doble-ciego, } \\
\text { activo } \\
\text { controlado } \\
\text { con placebo }\end{array}$ & Inglés & $\begin{array}{l}\text { Evaluar la adaptación } \\
\text { a los turnos de noche } \\
\text { en los médicos de } \\
\text { emergencia. }\end{array}$ & $\begin{array}{l}\text { No mejoró significativa- } \\
\text { mente el sueño, ( } 5.13 \\
\text { horas frente a } 6.20 \\
\text { horas, p<0,05), el esta- } \\
\text { do de ánimo o el rendi- } \\
\text { miento en la intubación } \\
\text { de un maniquí en los } \\
\text { trabajadores de turno } \\
\text { nocturno. Aunque en } \\
\text { los resultados del ECG } \\
\text { no se presentó diferen- } \\
\text { cias significativas entre } \\
\text { el turno de noche y el } \\
\text { turno de día. Sugirien- } \\
\text { do que las interrupcio- } \\
\text { nes del ritmo circadiano } \\
\text { es un problemas para } \\
\text { los médicos de } \\
\text { emergencia. }\end{array}$ \\
\hline
\end{tabular}

\subsection{Otros efectos sobre la salud del trabajador por turnos de trabajo y guardias nocturnas}

Los turnos de trabajo y las guardias pueden repercutir sobre la salud del médico. Entre los artículos revisados se detallan varios efectos sobre la salud del trabajador, algunos se limitan a mencionar que "la turnicidad afectan el ritmo circadiano y por tanto esto provoca efectos negativos".

Los horarios nocturnos en el personal médico pueden provocar desde un simple estado de ánimo negativo el día después de la guardia, como un estado total de Síndrome de Burnout o síndrome del trabajador quemado ${ }^{29,30}$.

El estrés elevado que en el ambiente sanitario se da por las continuas demandas ambientales que recibe el médico cuando consulta a uno, otro y otro paciente, y debe afrentarse dando una respuesta adecuada. Gloria $\mathrm{Kuhn}^{29}$ en su estudio menciona una encuesta realizada por "The American College of Emergency Physicians (ACEP)", donde 108 de los encuestados respondieron que el horario irregular es el aspecto más estresante del servicio de emergencia. 
Los turnos nocturnos son los más implicados en las alteraciones del sueño que es otro de los efectos. Kuhn ${ }^{29}$ menciona que la consecuencia más generalizada y grave de la desincronización del sueño, es la disminución en cantidad y calidad del mismo, ya que los ruidos y perturbaciones no permiten el descanso mientras se duerme de día, además de que el sueño diurno presenta menos periodos REM. También describe que los médicos residentes de varias especialidades se ven afectados por la falta de sueño. Por ejemplo, la alteración del sueño a los pediatras les afecta más la habilidad motora fina, a los internistas les desarrolla un deterioro significativo, los anestesiólogos pautan peor, mientras que los se aprecian resultados claros en los cirujanos, y es que trastorno de sueño de los turnos de trabajo, se produce cuando el individuo tiene que trabajar superponiendo su período de sueño normal, provocando una discrepancia entre el reloj biológico circadiano endógeno y su real descanso físico. Según la Clasificación Internacional del trastorno del sueño, para hacer referencia a un estado de somnolencia o de insomnio debe de ocurrir por lo menos durante un mes, clasificando en 3 grupos dichos trastornos en los trabajadores: 1) Aquellos trabajadores que no tienen discapacidad, 2) Aquellos trabajadores que tienen discapacidad pero que no cumplen los criterios del Departamento de Bienestar Social y 3) Aquellos trabajadores que si cumplen con los criterios de diagnostico del Departamento. Jonathan R.L. y Schwartz M.D. señalan que tanto los del grupo 2 y 3 de la clasificación internacional de trastornos del sueño, como no son capaces de satisfacer las demandas del trabajo, deciden cambiarse a trabajar en horarios diurnos o simplemente a renunciar o retirarse del trabajo a la fuerza ${ }^{31}$.

Las alteraciones del estado de alerta y rendimiento, evidenciado en la revisión de Kuhn ${ }^{29}$ que comenta los resultados de Moore-ede M, et al que estudiaron en un laboratorio de test psicomotor el rendimiento y la capacidad de resolver problemas matemáticos, llegaron a la conclusión de que entre las 3 am y las 5 am se encuentran niveles bajos de rendimiento. Saricaoglu $\mathrm{F}$ et al. ${ }^{32}$ en su estudio a través de un cuestionario pre y post jornadas laborales, detectó que los niveles más altos de ansiedad pueden conducir a un mayor deterioro en las funciones cognitivas del trabajador.

Las alteraciones cardiovasculares como en el estudio prospectivo aleatorizado doble ciego y cruzado de M. Rauchenzauner et al. ${ }^{33}$ donde fueron evaluados 40 médicos durante 24 horas, obtuvieron como resultado que los turnos nocturnos tienen un perfil de mayor riesgo para la enfermedad cardiovascular. Concretamente mostró que había una mayor tasa de latidos ventriculares prematuros (LVP) (0-6h, p=0.047), además de alteraciones en las tensiones arteriales, con una elevación en la presión sistólica durante el turno de noche en comparación con las tensiones arteriales de médicos de emergencia después de la guardia, sin olvidar que también se evidencio un aumento de los latidos cardiacos durante la jornada ${ }^{29}$. Se demostró en 40 médicos que durante un turno de $24 \mathrm{~h}$ la presión arterial (PA) diastólica fue mayor $(\mathrm{p}=0.025)$, mas aun, durante la noche se encontraba asociado a una elevada presión sistólica de más de $125 \mathrm{mmHg}$ durante el tiempo de sueño $(p=0.028)^{33}$. Otro estudio que trata del tema es el realizado por Adams et al. donde se evalúa a 12 residentes que presentan un aumento de 5,5 $\mathrm{mm} \mathrm{Hg}$ en su PA diastólica media con un IC 95\% durante la actividad del turno de noche. Además de que se observaron arritmias no diagnosticadas ${ }^{34}$.

Otros de los efectos que parecen encontrarse asociados se relacionan con las alteraciones gastrointestinales entre los cuales podemos citar dispepsia, constipación, diarrea, etc. ${ }^{30}$ Los abortos, efectos psiconeuroticos, efectos musculo esquelético son también mencionados por Feo J. ${ }^{30}$ Aumento del consumo de medicación para dormir o el uso de tranquilizantes ${ }^{29}$. El envejecimiento y el cáncer de mama que aun están en la prima fase de estudio ${ }^{30}$.

Concretamente 5 artículos refieren algunas medidas o estrategias para hacer más tolerable estos cambios de horarios en los trabajadores:

Durante la Guardia:

- Siestas o NAP de corto tiempo ${ }^{25,29}$.

- Consumo de café ( $4 \mathrm{mg} / \mathrm{kg}$ de peso) ${ }^{25,29}$ 
- Mejorar la luz artificial en el área de trabajo ${ }^{29}$.

- Variar la actividad sobre todos cuando se está aburrido ${ }^{28}$.

Después de la Guardia:

- Gestionar el sueño en un buen ambiente (cuarto oscuro, silencioso, utilizar tapones en los oídos, en una superficie confortable, con una temperatura agradable) $)^{25,28,29}$

- Dormir el mayor tiempo posible ${ }^{28,29}$.

- Realizar el camino a la casa usando gafas de sol, evitar la actividad diurna 25,28,29.

- Si se presenta hambre a la hora de dormir, comer algo ligero ${ }^{28}$.

- Evitar la cafeína al menos 4 horas antes de la hora de dormir ${ }^{28}$.

- Uso de melatonina para el tratamiento de las alteraciones circadianas(en estudio) ${ }^{29}$.

- El Colegio Americano de Médicos de Emergencia postula que la institución debe ofrecer un lugar para dormir antes de conducir a casa después de turnos de noche ${ }^{35}$.

En general:

- El Colegio Americano de Médicos de Emergencia: postula que los horarios deben se programados siempre que sea posible de manera coherente con los principios circadianos (mañana, tarde, noche) ${ }^{35}$.

- Los hospitales con médicos residentes deberían proveer al residente de un medio de trasporte de regreso a casa después de una guardia ${ }^{27}$.

- Entre guardias no debería programarse actividades extras ${ }^{29}$.

Tabla 4. Otros efectos que se producen en la salud de los médicos por los turnos de trabajo y las guardias.

\begin{tabular}{|c|c|c|c|c|c|c|}
\hline N. ${ }^{\circ}$. & Autor & Pub. & Tipo de estudio & Idioma & Efectos en la Salud & Resumen \\
\hline 1 & $\begin{array}{l}\text { Olson EJ; Drage } \\
\text { LA; Auger RR. } 25\end{array}$ & 2009 & $\begin{array}{l}\text { Descriptivo } \\
\text { Revision }\end{array}$ & Inglés & $\begin{array}{l}\text { Alteraciones del sueño, } \\
\text { del estado de alerta y } \\
\text { rendimiento, Medidas o } \\
\text { estrategias para tolerar } \\
\text { los trabajos por turnos. }\end{array}$ & $\begin{array}{l}\text { Descripción básica } \\
\text { de los procesos de } \\
\text { regulación del sueño, } \\
\text { del impacto de los } \\
\text { horarios, las pruebas } \\
\text { de laboratorio que } \\
\text { demuestran efectos } \\
\text { en los médicos y los } \\
\text { efectos personales por } \\
\text { dicha pérdida de sueño. }\end{array}$ \\
\hline 2 & $\begin{array}{l}\text { Blum AB, } \\
\text { Raiszadeh } \\
\text { F, Shea S, } \\
\text { Mermin, Lurie P, } \\
\text { Landrigan C, et } \\
\text { al. } 27\end{array}$ & 2010 & Transversal & $\begin{array}{l}\text { Inglés- } \\
\text { EE.UU. }\end{array}$ & $\begin{array}{l}\text { Medidas o estrategias } \\
\text { para tolerar los trabajos } \\
\text { por turnos }\end{array}$ & $\begin{array}{l}\text { Encuesta nacional } \\
\text { de } 1.200 \text { miembros } \\
\text { representativos de } \\
\text { la opinión pública a } \\
\text { través del teléfono } \\
\text { de marcación digital } \\
\text { al azar con el fin de } \\
\text { describir EE.UU. la } \\
\text { opinión pública sobre } \\
\text { la regulación de médico } \\
\text { residente horas de } \\
\text { trabajo, en particular } \\
\text { con referencia a las } \\
\text { recomendaciones } \\
\text { del IOM. }\end{array}$ \\
\hline
\end{tabular}




\begin{tabular}{llclcl}
\hline N. ${ }^{\circ}$. & \multicolumn{1}{c}{ Autor } & Pub. & Tipo de estudio & Idioma & \multicolumn{1}{c}{ Efectos en la Salud } \\
\hline 3 & Smith-Coggins & Oct & Prospectivo, & Inglés & Alteraciones del \\
& R; Rosekind & 1997 & doble-ciego, & & sueño, problemas \\
& MR; et al. 28 & & activo & & gastrointestinales \\
& & controlado & & y cardiovasculares, \\
& & con placebo & & Medidas o estrategias \\
& & & & para tolerar los trabajos \\
& & & & por turnos.
\end{tabular}

$4 \quad$ Kuhn G. 29 Ene
2001

Descriptivo

5 Feo J. 30

2008 Revisión

6 Jonathan R. L. Schwartz JRL. 31
2010 Artículo de revista
Inglés Alteraciones del

sueño, estrés elevado, alteraciones del estado de alerta y rendimiento, enfermedades cardiovasculares y gastrointestinales. Alteración de las tensiones arteriales, Medidas o estrategias para tolerar los trabajos por turnos.

Español Estrés elevado, Colom- alteraciones del

bia sueño, problemas gastrointestinales, enfermedades cardiovasculares, abortos, efectos psiconeuróticos.

Inglés Alteración del sueño (insomnio).
Describir como el trabajo por turnos afecta la salud y la vida cotidiana. Caracterizando los efectos ocasionadas por el trabajo por turnos, en la salud de las personas. Menciona los trastornos musculo-esqueléticos, abortos, problemas de sueño. Además de las alteraciones familiares, sociales y las principales estrategias para minimizar los efectos negativos.

Este artículo tiene como objetivo caracterizar los síntomas y factores de riesgo asociados con el tras-torno que genera los cambios en los turnos de trabajo en los médicos de atención primaria, con el fin de ayudar a los mismos en el diagnostico y reconocimien-to de este trastorno tan subestimado. 


\begin{tabular}{|c|c|c|c|c|c|c|}
\hline N. ${ }^{\circ}$. & Autor & Pub. & Tipo de estudio & Idioma & Efectos en la Salud & Resumen \\
\hline 7 & $\begin{array}{l}\text { Saricaoglu F, } \\
\text { Akinci SB, } \\
\text { Gozacan A, } \\
\text { Guner B, Rezaki } \\
\text { M, Aypar U. } 32\end{array}$ & 2005 & $\begin{array}{l}\text { Descriptivo } \\
\text { Transversal. }\end{array}$ & Inglés & $\begin{array}{l}\text { Trastornos del sueño, } \\
\text { ansiedad, fatiga, etc. }\end{array}$ & $\begin{array}{l}\text { Se aplicó un cuestionario } \\
\text { a todos los sujetos antes } \\
\text { y después de los turnos } \\
\text { en la primera jornada } \\
\text { de } 5 \text { días. Las funciones } \\
\text { cognitivas, la ansiedad, } \\
\text { pensamientos subjetivos } \\
\text { sobre el cansancio, } \\
\text { el estrés, la duración } \\
\text { del sueño y la calidad } \\
\text { se evaluaron en este } \\
\text { cuestionario. Se concluyó } \\
\text { que los niveles más } \\
\text { altos de ansiedad puede } \\
\text { conducir a un mayor } \\
\text { deterioro en las funciones } \\
\text { cognitivas. }\end{array}$ \\
\hline 8 & $\begin{array}{l}\text { Rauchenzauner } \\
\text { M; Ernst F; } \\
\text { Hintringer F; } \\
\text { Ulmer H; et } \\
\text { al. } 33\end{array}$ & $\begin{array}{l}\text { Nov } \\
2009\end{array}$ & $\begin{array}{l}\text { Prospectivo } \\
\text { aleatorizado } \\
\text { doble ciego } \\
\text { y cruzado }\end{array}$ & $\begin{array}{l}\text { Inglés- } \\
\text { Australia }\end{array}$ & $\begin{array}{l}\text { Enfermedades } \\
\text { cardiovasculares, estrés } \\
\text { elevado, alteraciones de } \\
\text { las tensiones arteriales. }\end{array}$ & $\begin{array}{l}\text { Estudio hecho en } \\
\text { Australia a } 44 \text { médicos, } \\
\text { durante las guardias para } \\
\text { evaluar los efectos en el } \\
\text { ECG, la variabilidad del } \\
\text { ritmo cardiaco y la tensión } \\
\text { arterial. Marcadores de } \\
\text { estrés en el suero o la } \\
\text { orina. Concluyendo que } \\
\text { estos turnos aumentan los } \\
\text { riesgo cardiovasculares. }\end{array}$ \\
\hline 9 & $\begin{array}{l}\text { Adams SL; Roxe } \\
\text { DM; Weiss } \\
\text { J; Zhang F; } \\
\text { Rosenthal JE } 34\end{array}$ & $\begin{array}{l}\text { Sep } \\
1998\end{array}$ & Prospectivo & Inglés & $\begin{array}{l}\text { Enfermedades } \\
\text { cardiovasculares, } \\
\text { alteraciones de las } \\
\text { tensiones arteriales. }\end{array}$ & $\begin{array}{l}\text { Busca determinar los } \\
\text { efectos fisiológicos de } \\
\text { los turnos de trabajo } \\
\text { nocturnos en los } \\
\text { médicos. Valorando } \\
\text { las presión arterial y } \\
\text { variabilidad cardiaca. } \\
\text { Demostrándose que } \\
\text { el tono simpático esta } \\
\text { elevado tanto antes } \\
\text { como durante las } \\
\text { guardias. Y que la PA se } \\
\text { modifica por el estrés. }\end{array}$ \\
\hline 10 & $\begin{array}{l}\text { Guía de } \\
\text { American } \\
\text { College } 35\end{array}$ & 1995 & Guía & Inglés & $\begin{array}{l}\text { Medidas o estrategias } \\
\text { para tolerar los trabajos } \\
\text { por turnos. }\end{array}$ & $\begin{array}{l}\text { Guía aprobada } \\
\text { inicialmente por } \\
\text { el Consejo de } \\
\text { Administración ACEP } \\
\text { septiembre 1994, Junta } \\
\text { de Directores 1998. Y } \\
\text { revisada y aprobada } \\
\text { por ACEP Consejo } \\
\text { de Administración } \\
\text { septiembre de } 2003 \text { y } \\
\text { junio } 2010 \text {. }\end{array}$ \\
\hline
\end{tabular}




\section{En el personal de enfermería}

El personal de enfermería es otro de los sectores de salud que también se ve afectado por la turnicidad. En el estudio de H. Fukuda et al. ${ }^{36}$ describen los problemas que puedan presentarse en una enfermera con un turno de trabajo de 16 o de 8 horas, a partir de un estudio de casos y controles compuesto por 20 enfermeras cada uno y en el que no encuentran diferencia significativa en los síntomas de cada grupo.

Romana Albaladejo et al. ${ }^{37}$ realizaron un estudio en un Hospital de Madrid con una población de 622 profesionales, de los cuales el 58,7\% trabajaban como personal de enfermería y el 41,3\% como auxiliares/técnicos.

Elaboraron un cuestionario por medio del cual se recogieron las variables sociodemográficas (edad, sexo, categoría profesional, servicio, estado civil número de hijos, antigüedad en la profesión y en el puesto de trabajo, situación y turno laboral, número de enfermos a su cargo). Estas preguntas se valoraron según la escala de Likert con una puntuación de 1 a 5 , de forma que las puntuaciones inferiores a 3 traducían sentimientos positivos, 3 indicaba un nivel intermedio y valores superiores a 3 expresaban respuestas negativas respecto a las cuestiones planteadas. La segunda herramienta empleada en la recogida de la información fue el Maslach Burnout Inventory (MBI), en su versión validada en español.

El personal de enfermería está más despersonalizado $(\mathrm{p}=0,004)$ y menos realizado $(\mathrm{p}=0,036)$ que el grupo de auxiliares/técnicos. Cuando se analizaron los resultados de las 4 escalas (cansancio emocional, despersonalización, realización personal y Burnout) por servicios comprobamos que el agotamiento es superior en los trabajadores de oncología y urgencias $(p=0,001)$, la despersonalización en urgencias $(p=0,007)$, y el Burnout es de nuevo más alto en las áreas como oncología y urgencias $(\mathrm{p}=0,000)$. Los profesionales que respondían que su labor asistencial era poco reconocida, obtenían las peores puntuaciones en el Burnout y sus tres dimensiones (cansancio emocional, despersonalización, realización personal) ( $\mathrm{p}=0,000)$. A menor grado de satisfacción laboral más altas son las puntuaciones en las 4 escalas (cansancio emocional, despersonalización, realización personal y Burnout $)(\mathrm{p}=0,000)^{37}$.

Statistics Canadá en colaboración con el Instituto Canadiense de Información de Salud y Sanidad de Canadá, realizó una encuesta nacional en este país sobre el trabajo y la salud de las enfermeras, durante el periodo comprendido entre octubre 2005 hasta enero 2006. En el mismo se detectó que casi una quinta parte (19\%), de las enfermeras de un Hospital de Canadá, había administrado medicación por error, a los pacientes bajo su cuidado, lo cual se había producido frecuentemente u ocasional, en el año 2005. Las causas más importantes de estos errores fueron los siguientes; turno de trabajo nocturno, carga de trabajo, insatisfacción en el trabajo, etc. ${ }^{38}$

Las enfermeras que trabajan turnos diferentes pueden mejorar la tolerancia al trabajo por turnos cuando aprenden a adoptar medidas para reducir las variaciones en el ritmo sueño-vigilia. Por ejemplo, ajustar los ritmos internos, mejorar el sueño durante el día, mejorar el funcionamiento familiar y reducir la somnolencia en el trabajo para así disminuir los errores. Entre las medidas más efectivas se mencionan dormir la siesta, la eliminación de horas extras en turnos de 12 horas, completar las tareas difíciles de atención al paciente antes de las 4 am. ${ }^{39}$

En un estudio realizado en un Hospital de Brasil se detectó una diferencia significativa entre la calidad percibida del sueño diurno en comparación con el sueño nocturno del personal de enfermería y auxiliares ${ }^{40}$.

En relación al personal de enfermería de cuidados intensivos, un estudio de Ríos Risquez et al $2008^{41}$, encontró que estas son más vulnerables a presentar elevados niveles de agotamiento emocional, siendo los enfermeros de género masculino los más propensos a las actitudes de despersonalización. 
Tabla 5. Efectos de los turnos de trabajo en el personal de enfermería.

\begin{tabular}{|c|c|c|c|c|}
\hline N. ${ }^{\circ}$ & Autor & Pub. & Tipo de estudio & Resumen \\
\hline 1 & $\begin{array}{l}\text { Fukuda H, } \\
\text { Takahashi M, } \\
\text { Miki K, Haratani } \\
\text { T, Kurabayashi } \\
\text { L, et al.36 }\end{array}$ & $\begin{array}{l}\text { Abril } \\
1999\end{array}$ & $\begin{array}{l}\text { Ensayo de } \\
\text { campo }\end{array}$ & $\begin{array}{l}\text { Evalúa los problemas asociado a los turnos de trabajo en } \\
\text { enfermería comparando la interacción del ritmo circadia- } \\
\text { nos, un cuestionario la actividad física y postura en } 20 \\
\text { enfermeras con } 16 \mathrm{~h} \text { de trabajo y } 20 \text { con solo } 8 \mathrm{~h} \text {. }\end{array}$ \\
\hline 2 & $\begin{array}{l}\text { Albaladejo R, } \\
\text { Villanueva R, } \\
\text { Ortega P, Astasio } \\
\text { P, Calle ME, } \\
\text { Dominguez V. } 37\end{array}$ & 2004 & Transversal & $\begin{array}{l}\text { Se concluyó que en estudio el perfil del sujeto afecto del } \\
\text { burnout se corresponde con el de un profesional con ex- } \\
\text { periencia laboral que sin embargo manifiesta un escaso } \\
\text { grado de reconocimiento a su labor asistencial y un alto ni- } \\
\text { vel de insatisfacción con la gestión de su centro de trabajo. }\end{array}$ \\
\hline 3 & $\begin{array}{l}\text { Wilkins K, } \\
\text { Shields M } 38\end{array}$ & 2008 & Transversal & $\begin{array}{l}\text { Se evidencio que aproximadamente un } 19 \% \text { de las enfer- } \\
\text { meras de un hospital de Canadá que laboran en diferen- } \\
\text { tes aéreas, administro medicación errónea a pacientes y } \\
\text { que ocurrió de forma frecuente u ocasional. Las causas } \\
\text { más importantes son las siguientes; turno de trabajo noc- } \\
\text { turno, carga de trabajo, insatisfacción en el trabajo, etc. }\end{array}$ \\
\hline 4 & $\begin{array}{l}\text { Berger A, } \\
\text { Hobbs B } 39\end{array}$ & 2006 & $\begin{array}{l}\text { Artículo de } \\
\text { Revista. }\end{array}$ & $\begin{array}{l}\text { Analiza las consecuencias que tienen los turnos rotativos } \\
\text { y las guardias nocturnas sobre el ritmo circadiano, ritmo } \\
\text { sueño vigilia, efectos psicológicos, la seguridad para el } \\
\text { paciente, y la tolerancia al trabajo en el personal de en- } \\
\text { fermería. }\end{array}$ \\
\hline 5 & $\begin{array}{l}\text { Da Silva Borges } \\
\text { FN, Fischer FM. } \\
40\end{array}$ & 2003 & $\begin{array}{l}\text { Descriptivo } \\
\text { Transversal. }\end{array}$ & $\begin{array}{l}\text { Se evaluó el impacto de las } 12 \text { horas del turno de noche } \\
\text { fijo (19:00-07:00 h) de trabajo, seguido de } 36 \mathrm{~h} \text { de plantas } \\
\text { fuera de tiempo, en el ciclo sueño-vigilia, la duración del } \\
\text { sueño, la percepción subjetiva de la calidad del sueño, y } \\
\text { estado de alerta en tiempo de trabajo en un grupo com- } \\
\text { puesto por } 5 \text { enfermeras y 15auxiliares de enfermería. }\end{array}$ \\
\hline 6 & $\begin{array}{l}\text { Ríos Risquez } \\
\text { MI, Godoy } \\
\text { Fernández } \\
\text { C, Peñalver } \\
\text { Hernandez F, } \\
\text { Alonso Tovar AR, } \\
\text { López Alcaraz F, } \\
\text { López Romera A, } \\
\text { et al. } 41\end{array}$ & 2008 & $\begin{array}{l}\text { Descriptivo } \\
\text { Transversal }\end{array}$ & $\begin{array}{l}\text { Se comparó y estimó el nivel de burnout existente en los } \\
\text { Servicios de cuidados Intensivos y Urgencias del Hospital } \\
\text { Morales Meseguer. Se estudió la asociación de burnout } \\
\text { con características sociodemográficos y laborales de los } \\
\text { profesionales de enfermería encuestados. Los niveles en- } \\
\text { contrados de burnout resultaron ser moderados-altos. Los } \\
\text { profesionales del área de cuidados intensivos son los mas } \\
\text { vulnerables a padecer elevados niveles de agotamiento } \\
\text { emocional y el género masculino es el más propenso a } \\
\text { las actitudes de despersonalización. }\end{array}$ \\
\hline
\end{tabular}

\section{CONCLUSIONES}

Las largas jornadas de trabajo, servicios de guardia durante la noche y la rotación de turnos, son características implícitas de la práctica médica hospitalaria que no se pueden hacer desaparecer de la práctica diaria, por lo que queda en manos de la medicina de trabajo velar por el trabajador y su salud en relación a los posibles efectos adversos y las repercusiones que estas condiciones puedan ejercer sobre los trabajadores, mediante la vigilancia de la salud y la prevención frente a riesgos inherentes.

La identificación de los factores de riesgo psicosocial a los que pueden estar expuestos los médicos y enfermeras permitirá adoptar medidas preventivas, que pueden ser útiles para mejorar la salud y la calidad de vida de estos profesionales. Se debe insistir en que la programación de las guardias sean realizadas y dadas a conocer con antelación y con tiempos adecuados para que pueda producirse la recuperación, ya que se ha demostrado que estas medidas contribuyen a disminuir el estrés y permiten otorgar 
mayor autonomía del tiempo, tanto al médico como a la enfermera. Hecho de suma importancia, ya que algunos profesionales, debido a las consecuencias de los turnos de trabajo, pueden abandonar el ejercicio o enfermar.

\section{REFERENCIAS BILIOGRÁFICAS}

1. Amelsvoort LG. Impact of one year of shift work on cardiovascular disease risk factors. J Ocupp Envor Med 01 jul 2004; 46(7): 699-706.

2. Waterhouse JM. Shift work. BMJ; 1994: 308(6944): 1640, [citado el 12 enero 2011] http://www.ncbi.nlm. nih.gov/pmc/articles/PMC2540445/pdf/bmj00445-0064c.pdf

3. Smith-Coggins R. et al. Rotating Shiftwork schedules: Can we enhance physician adaptation to nigth shifts?. Academic Emergency medicine; 1997: 4(10): 951-61.

4. Moore-EdeMC, Richardson GS. Medical implications of shift-work. Annu Rev Med. 1995; 36:607-17.

5. Mingote Adán JC. Síndrome "burnout». Síndrome de desgaste profesional. Monografías de Psiquiatría 1977; 5: 1-44.

6. Moreno-Jiménez B, González JL, Garrosa E. Desgaste profesional (burnout), personalidad y salud percibida. En: Buendía J, Ramos F. Empleo, estrés y salud. Madrid: Psicología Pirámide; 2001

7. Gil-Monte PR, Peiró JM. Un estudio comparativo sobre criterios normativos y diferenciales para el diagnostico del síndrome de quemarse por el trabajo (burnout) según el MBI-HSS en España. Revista de Psicología del Trabajo y de las Organizaciones. 2000; 16 (2): 135-149.

8. Freudenberger HJ. Staff Burn-out. J Soc Issues. 1974; 30:159-165.

9. Maslach C, Jackson SE. Maslach Burn-out Inventory. Palo Alto: Consulting Pysichologists Press; 1986.

10. Mingote JC. Síndrome burnout o síndrome de desgaste profesional. FMC. 1998; 5: 493-503.

11. Pines A, Guendelman S. Exploring the relevance of burnout to mexican blue collar women. J Vocat Behav. 1995; 47: 1-20.

12. Gil-Monte PR. El síndrome de quemarse por el trabajo (burnout) como fenómeno transcultural. Información Psicológica. 2008; 91-92: 4-11.

13. Dr. Antonio Cano Vindel. Sociedad Española para el estudio de la ansiedad y el estrés (SEAS). La naturaleza del estrés. [citado el 22 enero 2011] http://www.ucm.es/info/seas/estres_lab/el_estres.htm

14. McEvoy RD; Lack LL, Medical staff working the night shift: can naps help? Med J Aust; 2006: 185(7): $349-50$.

15. MA Caballero Martín a, F Bermejo Fernández a, R Nieto Gómez a, F Caballero Martínez a. Prevalencia y factores asociados al Burnout en un área de salud. Atención Primaria 2001, Vol.27 Núm. 05:313-7. ISSN:0212-6567.

16. Escribà-Agülr V, Artazcoz L, Pérez-Hoyos S. Efecto del ambiente psicosocial y de la satisfacción laboral en el síndrome de burnout en médicos especialistas. Gac. Sanit. (Esp). 2008; 22(4):300-8.

17. Armand Grau D, Suñer R, Prats M, Braga F. Influencia de los factores personales, profesionales y transnacionales en el Síndrome de Burnout en Personal Sanitario Hispanoamericano y Español. Rev Esp Salud Pública 2009; 83: 215-230.

18. Sánchez- Madrid MA, Delgado Martínez AD, Alcalde Pérez D. Prevalencia del síndrome de burnout o desgaste profesional en los cirujanos ortopédicos de España. Revista Ortopédica Traumatología. Madrid 2005. 49 (5):364-367.

19. Cebriá J, Palma C, Sobrequés J, Ger S, Ferrer M, Segura J. Evolución del síndrome de desgaste profesional en médicos de familia: estudio longitudinal a 5 años. Semergen. 2008; 34 (3): 107-112.

20. Fonseca M, Sanclemente G, Hernandez C, Visiedo C, Bragulat E, Miró O. Residentes, guardias y síndrome de Burnout. Rev Clin Esp. 2010; 210(5):209-215.

21. Fernández Martínez O, Hidalgo Cabrera C, Martin Tapia A, Montero Suarez S, García del Rio García B. Burnout en médicos residentes que realizan guardias en un servicio de urgencias. Emergencias (Sant Vicenç dels Horts). 2007; 19 (3): 116-121.

22. Casado Maragón A., Muñoz Duran G., Ortega González N., Castellanos Asenjo A. Rodríguez Blázquez ME, García Aroca C. Burnout y factores asociados en profesionales del Hospital Gregorio Marañón de Madrid. Med Segur Trab. 2005: 51(201): 27-36. 
23. Frade Mera MJ, Vinagre Gaspar R, Zaragoza García I, Viñas Sánchez S, Antúnez Melero E, Álvarez González S, et al. Síndrome de burnout en distintas Unidades de Cuidados Intensivos. Enferm. Intensive 2009; 20 (4): 131-140.

24. Lockley SW; Landrigan CP; Barger LK; Czeisler CA+ Harvard Work Hours Health and Safety Group. When policy meets physiology: the challenge of reducing resident work hours. Clin Orthop Relat Res; 2006: 449: 116-27.

25. Olson EJ; Drage LA; Auger RR. Sleep deprivation, physician performance, and patient safety. Chest; 2009: 136(5): 1389-96

26. Wilhelm BJ; Widmann A; Durst W; Heine C; Otto G. Objective and quantitative analysis of daytime sleepiness in physicians after night duties. Int J Psychophysiol; 2009: 72(3): 307-13.

27. Blum AB; Raiszadeh F; Shea S; Mermin D; Lurie P; Landrigan CP; Czeisler CA. US public opinion regarding proposed limits on resident physician work hours. BMC Med; 2010: 8: 33.

28. Smith-Coggins R; Rosekind MR; Buccino KR; Dinges DF; Moser RP. Rotating shiftwork schedules: can we enhance physician adaptation to night shifts? Acad Emerg Med; 1997: 4(10): 951-61.

29. Kuhn G. Circadian rhythm, shift work, and emergency medicine. Ann Emerg Med; 2001, 37(1): 88-98.

30. Feo J. Influencia del Trabajo por turnos en la salud y la vida cotidiana. Rev de la Pontificia Universidad Javeriana de Bogota. 2007.

31. Jonathan R.L. Schwartz, MD. Recognition of shift-Work disorder in primary care Supplement to The Journal of Family Practice. 2010: 59 (1): S18-S23.

32. Saricaoglu F, Akinci SB, Göza冈an A., Güner B, Rezaki M., Aypar Ü. The effect of day and night shift working on the attention and anxiety levels of anesthesia. USA. 2005: 16(2); 116-112.

33. Rauchenzauner M; Ernst F; Hintringer F; Ulmer H; Ebenbichler CF; Kasseroler MT; Joannidis M Arrhythmias and increased neuro-endocrine stress response during physicians' night shifts: a randomized cross-over trial. Eur Heart J; 2009 : 30(21): 2606-13.

34. Adams SL; Roxe DM; Weiss J; Zhang F; Rosenthal JE. Ambulatory blood pressure and Holter monitoring of emergency physicians before, during, and after a night shift. Acad Emerg Med; 1998: 5(9): 871-7.

35. Guía de American College. Emergency physician shift work. American College of Emergency Physicians. Ann Emerg Med; 1995: 25(6): 864

36. Fukuda H, Takahashi M, Miki K, Haratani T, Kurabayashi L, Hisanaga N, Arito H, Takahashi H, Egoshi M, Sakurai M. Shift work-related problems in 16-h night shift nurses (1): Development of an automated data processing system for questionnaires, heart rate, physical activity and posture. Ind Health. 1999 Apr;37(2):219-27.

37. Albaladejo R, Villanueva R, Ortega P, Astasio P, Calle ME, Dominguez. Síndrome de burnout en el personal de enfermería de un hospital de Madrid. Rev Clin Esp. 2004; 78(4).

38. Wilkins K, Shields. Corretales of medication error in hospitals. Health Report. 2008: 19 (2); Catalogue 82-003.

39. Berger AM, Hobbs BB. Impact of shift work on the health and safety of nurses and patients. Clinical Journal of Oncology Nursing. 2006; 10 (4): 465-471.

40. Da Silva Borges FN, Fischer FM. Twelve-Hour Night Shifts of Healthcare Workers: A Risk to the Patients? Department of Environmental Health, School of Public Health. Brasil.2003; 20. (2):351-360.

41. Ríos Risquez MI, Godoy Fernández C, Peñalver Hernandez F, Alonso Tovar AR, López Alcaraz F, López Romera A, et al. Estudio comparativo del burnout en personal de enfermería de Cuidados Intensivos y Urgencias. Enferm intensiva. 2008. 19(1): 2-13. 